\title{
Two-body Coulomb problems with sources for the J-matrix method
}

\author{
L. U. Ancarani ${ }^{a, *}$ and G. Gasaneo ${ }^{b}$ \\ ${ }^{a}$ Laboratoire de Physique Moléculaire et des Collisions, Université Paul Verlaine-Metz, \\ 57078 Metz, France \\ ${ }^{b}$ Departamento de Física, Universidad Nacional del Sur and Consejo Nacional de \\ Investigaciones Científicas y Técnicas, 8000 Bahía Blanca, Buenos Aires, Argentina
}

Received 11 December 2010; Accepted (in revised version) 26 December 2010

Published Online 28 March 2011

\begin{abstract}
The two-body Coulomb problem with sources is studied. Closed form solutions are provided for particular non-homogeneities used within the J-matrix method. The results are employed to build analytical solutions having incoming, outgoing and standing wave asymptotic conditions.
\end{abstract}

PACS: 03.65.Nk, 34.10.+x

Key words: coulomb problem, sources, J-matrix, non-homogeneous, hypergeometric

\section{Introduction}

Scattering theory is of fundamental importance in atomic physics. It allows for a description and an interpretation of many atomic collision processes such as, e.g., excitation and ionization by particle or radiation impact, among many others. In many cases, the wave function satisfying the full (time-independent) Schrödinger equation may be written as the sum of a known initial state of a simplified hamiltonian and an unknown scattering solution which describes the dynamics of the collision $[1,2]$. This separation leads straightforwardly to a non-homogeneous (driven) equation where the source is the product of the neglected interactions and the asymptotic solution. For example, this is the line followed by the Exterior Complex Scaling approach [3-5], which has been widely and successfully used in treating a large variety of scattering problems.

In a previous paper [6], we studied a two-body driven Schrödinger equation which includes a Coulomb interaction, a case of fundamental importance for the atomic physics community. Here, we shall apply some of the closed form results to the construction of an asymptotical cosine-like stationary function used by the J-matrix approach [7-9].

\footnotetext{
${ }^{*}$ Corresponding author. Email address: ancarani@univ-metz.fr (L. U. Ancarani)
} 
The J-matrix has proven to be a very successful method to treat a large variety of scattering problems, as reviewed by Alhaidari et al. [7]. It has been formulated for two-body problems without $[10]$ and with $[8,11]$ Coulomb interaction, but also for multichannel processes [1012]. The method can be considered as a variant of the variational approach for continuum states [13]. It bears some analogy with the R-matrix theory which divides the coordinate space into two regions, an inner region and an asymptotic region. The main idea of the J-matrix method is to separate the wave function into two parts: (i) an internal term which contains the information about the dynamics of the problem associated to a given potential $V$, and (ii) a term containing the asymptotic solution of the problem. The first part depends upon a large number of parameters which are used to solve the Schrödinger equation in the reaction zone where the interaction potential $V$ is not negligible. This term is usually expanded in a complete and orthogonal basis set. The second - asymptotic - part, on the other hand, ignores $V$ and is written as a linear combination $\varphi_{s}(r)+\tan \delta \varphi_{c}(r)$ of two stationary functions which at large distances $r$ from the origin behave as free particle - or Coulomb - waves with sinelike $\left(\varphi_{s}\right)$ and cosine-like $\left(\varphi_{c}\right)$ behaviors. This linear combination involves the coefficient $\tan \delta$ usually associated to the transition matrix of the scattering problem. The transition matrix and the expansion coefficients are determined by enforcing the proposal to satisfy the Schröginger equation.

The two functions $\varphi_{s}$ and $\varphi_{c}$ used to represent the asymptotic part must be linearly independent functions satisfying a Schrödinger equation which does not contain the interaction potential $V$. However, a problem arises: for a given radial Schrödinger equation, two solutions with the asymptotic behavior of $\varphi_{s}$ and $\varphi_{c}$, and being both regular at the origin, are not available. Thus the J-Matrix method makes use of a function $\varphi_{c}$ which, having cosine-like behavior, satisfies a non-homogeneous Schrödinger equation; its expansion on $L^{2}$ Laguerre basis functions was proposed, e.g., in Refs. $[8,11,12]$. The primary purpose of this paper is to give a closed form solution for $\varphi_{c}$.

The paper is arranged as follows. In Sec. II, we provide in closed form the particular solution for the two-body Coulomb Schrödinger equation with a particular non-homogeneity. This involves a two-variable hypergeometric function which can be considered as a generalization of the Kummer function associated to the pure Coulomb problem. In Sec. III, we give the asymptotic behavior of this solution, as well as that of the regular and irregular homogeneous solutions. We then use these results to construct, through an appropriate linear combination, pure incoming, outgoing or cosine-like waves which can be used within the J-matrix method. Atomic units are used throughout.

\section{The two-body Coulomb problem with a particular source}

The J-matrix method makes use of a function $\varphi_{c}$ which is solution of the following radial non-homogeneous equation $[8,12]$

$$
\left[-\frac{1}{2 \mu}\left(\frac{d^{2}}{d r^{2}}-\frac{l(l+1)}{r^{2}}\right)+\frac{z_{1} z_{2}}{r}-E\right] h_{l, \sigma}(r)=a_{l, \sigma} e^{-\lambda r} r^{l+\sigma},
$$


where the energy $E=k^{2} /(2 \mu)$ will be taken hereafter as positive (scattering states), and two charges $z_{1}$ and $z_{2}$ are interacting through a Coulomb potential; let $\alpha=z_{1} z_{2} \mu / k$ denote the Sommerfeld parameter where $\mu$ is the reduced mass. In Eq. (1), $l$ is the angular momentum, $\lambda$ is chosen as a real positive parameter, $\sigma$ is a non-negative integer, and $a_{l, \sigma}$ is an arbitrary constant. The general solution of Eq. (1), $h_{l, \sigma}^{G}(r)$, can be written as a sum of the three terms

$$
h_{l, \sigma}^{G}(r)=A_{l}^{\text {Reg }} v_{l}^{\text {Reg }}(r)+A_{l}^{\text {Irreg }} v_{l}^{\text {Irreg }}(r)+h_{l, \sigma}^{P}(r) .
$$

The first two, $v_{l}^{\text {Reg }}(r)$ and $v_{l}^{\text {Irreg }}(r)$, are, respectively, the regular and irregular solutions of the corresponding homogeneous equation, which is the well-known Coulomb differential equation $[2,14]$.

In Ref. [6] we studied Eq. (1) and provided a closed form for its particular solution $h_{l, \sigma}^{P}(r)$. It can be expressed as follows

$$
\begin{aligned}
h_{l, \sigma}^{P}(r)=-2 \mu a_{l, \sigma} & \frac{1}{(\sigma+1)(2 l+2+\sigma)} e^{i k r} r^{l+\sigma+2} \\
& \times \Theta^{(1)}\left(\begin{array}{c}
\sigma+1,1 \mid 2 l+2+\sigma, i \alpha+l+2+\sigma \\
i \alpha+l+2+\sigma \mid 2+\sigma, 2 l+3+\sigma
\end{array} \mid ;-(i k+\lambda) r,-2 i k r\right),
\end{aligned}
$$

where $\Theta^{(1)}$ represents a two-variable, Kampé de Fériet, hypergeometric function; its primary power series representation reads [15]

$$
\Theta^{(1)}\left(\begin{array}{c}
a_{1}, a_{2} \mid b_{1}, b_{2} \\
c_{1} \mid d_{1}, d_{2}
\end{array} \mid ; x_{1}, x_{2}\right)=\sum_{m_{1}=0}^{\infty} \sum_{m_{2}=0}^{\infty} \frac{\left(a_{1}\right)_{m_{1}}\left(a_{2}\right)_{m_{2}}\left(b_{1}\right)_{m_{1}}\left(b_{2}\right)_{m_{1}+m_{2}}}{\left(c_{1}\right)_{m_{1}}\left(d_{1}\right)_{m_{1}+m_{2}}\left(d_{2}\right)_{m_{1}+m_{2}}} \frac{x_{1}^{m_{1}} x_{2}^{m_{2}}}{m_{1} ! m_{2} !} .
$$

Other formulations (as series or integral representations) exist [15]. From a particular series representation (see Ref. [6]), the asymptotic behavior of $h_{l, \sigma}^{P}(r)$ can be extracted and written in a simple form (see next section).

At this point we should mention that the particular solution can also be obtained by making use of the partial wave Coulomb Green function $G_{C, l}\left(r, r^{\prime}\right)$ (see, e.g., [16]), through

$$
h_{l, \sigma}^{P}(r)=\int_{0}^{\infty} G_{C, l}\left(r, r^{\prime}\right)\left[a_{l, \sigma} e^{-\lambda r^{\prime}}\left(r^{\prime}\right)^{l+\sigma}\right] d r^{\prime} .
$$

It is normally stated that one theoretical advantage related to this approach is that it formally provides the appropriate asymptotic behavior of the solutions [1,2]. As can be shown in the present case, the actual calculation of (5) leads to an alternative series representation of $h_{l, \sigma}^{P}(r)$ which numerically provides the appropriate asymptotic limit. However, it is not obvious how to extract from it a simple closed form expression for the asymptotic behavior. Moreover, as shown by the authors [17], the Green function approach can lead to erroneous conclusions when dealing with long-range Coulomb interactions and inappropriate initial states. 


\section{Wave functions with different asymptotic behaviors}

3.1 Properties of the functions $v_{l}^{\text {Reg }}, v_{l}^{\text {Irreg }}$ and $h_{l, \sigma}^{P}$

Both solutions of the homogeneous equation, $v_{l}^{\text {Reg }}(r)$ which behaves as $r^{l+1}$ for $r \sim 0$ (regular) and $v_{l}^{\text {Irreg }}(r)$ which behaves as $r^{-l}$ for $r \sim 0$ (irregular), are real functions. The (asymptotic unit flux) Coulomb wave functions possess the following well-known large distances behaviors $[18]$

$$
\begin{aligned}
& v_{l}^{\text {Reg }}(r) \longrightarrow \sin \left[\Phi_{C}(l)+\sigma_{C}(l)\right], \\
& v_{l}^{\text {Irreg }}(r) \longrightarrow \cos \left[\Phi_{C}(l)+\sigma_{C}(l)\right],
\end{aligned}
$$

where

$$
\begin{aligned}
& \Phi_{C}(l)=k r-\alpha \ln (2 k r)-\frac{\pi}{2} l, \\
& \sigma_{C}(l)=\operatorname{Arg}[\Gamma(i \alpha+l+1)] .
\end{aligned}
$$

The term $\ln (2 \mathrm{kr})$ is the characteristic logarithmic distortion due to the long range of the Coulomb potential.

From expression (3), it is clear that the particular solution $h_{l, \sigma}^{P}(r)$ of the non-homogeneous differential equation (1) is regular at the origin. As shown by the authors [6], the function is real as long as the $a_{l, \sigma}$ is real, although this does not appear straightforwardly from expression (3). By studying the mathematical properties of the $\Theta^{(1)}$ hypergeometric function [19], and using a special series representation (Eq. (24) of [6]), the asymptotic behavior of $h_{l, \sigma}^{P}(r)$ has been shown [6] to have the following cosine behavior

$$
h_{l, \sigma}^{P}(r) \longrightarrow N_{\text {source }} \cos \left[\Phi_{C}(l+\sigma+2)+\delta(l, \sigma, \lambda)\right],
$$

with

$$
\begin{aligned}
& N_{\text {source }}=a_{l, \sigma} \mathscr{N}_{\text {source }} \\
& \mathscr{N}_{\text {source }}=-2 \mu \frac{2(1)_{\sigma} \Gamma(2 l+\sigma+2)}{(2 k)^{l+\sigma+2}|\Gamma(i \alpha+l+\sigma+2)|}\left|{ }_{2} F_{1}\right| e^{\frac{\pi}{2} \alpha}, \\
& { }_{2} F_{1}\left(\begin{array}{c}
\sigma+1,2 l+2+\sigma \\
i \alpha+l+2+\sigma
\end{array} \mid ; \frac{1}{2}\left(1+\frac{\lambda}{i k}\right)\right)=\left|{ }_{2} F_{1}\right| e^{i \Delta(l, \sigma, \lambda)}, \\
& \delta(l, \sigma, \lambda)=\sigma_{C}(l+\sigma+1)-\Delta(l, \sigma, \lambda),
\end{aligned}
$$

and where the functions $\Phi_{C}(l)$ and $\sigma_{C}(l)$ are defined by (7) and (8), respectively.

The non-homogeneous Schrödinger equation (1) is stationary. This means that a time independent flux of particle is observed in the system. The particular solution, given by Eq. (3), has the cosine-like behavior (9), thus expressing the stationary character of the flux. 
The appearing phase-shift is entangled in a complicated way as it depends on the different ingredients of the source. The effect of the exponential parameter $\lambda$ appears only in the Gauss function (10c). The power index $\sigma$, on the other hand, appears in all the terms building the phase-shift. This shows that it is not possible to disentangle the individual effects of both contributions of the source included in the non-homogeneous equation (1).

While the prefactor $N_{\text {source }}$ depends on the source itself through $\lambda, \sigma$ and $a_{l, \sigma}$, we could introduce some normalization in such a way that the solution $h_{l, \sigma}^{P}(r)$ behaves at large values of the coordinates as a pure cosine function with unit amplitude. This is discussed in the next subsection.

\subsection{The J-matrix wave functions}

As mentioned in the Introduction, the J-matrix method requires wave functions having pure Coulomb cosine-like $\left(\varphi_{c}\right)$ and sine-like $\left(\varphi_{s}\right)$ behavior at large distances. So far, the method has always been presented using Laguerre expansions for both functions. In Ref. [8], Yamani and Reinhardt presented a comparison between the Coulomb wave function $v^{R e g}(r)$ and its expansion in terms of Laguerre functions; they gave analytic expressions for the coefficients. No analytic expression for the function $\varphi_{c}$ having pure cosine-like behavior was provided aside from that resulting from the Laguerre expansion. The aim of this paper is to give closed form expressions not only for the function having cosine-like behavior, but also functions having pure incoming and outgoing behaviors at large distances $r$.

With this purpose in mind, we can set the constants of the general solution $h_{l, \sigma}^{G}(r)$ given by (2) to provide the desired behavior. First of all, $A_{l}^{\text {Irreg }}$ is set equal to zero to avoid the irregular behavior of $v_{l}^{\text {Irreg }}(r)$ at the origin. To choose the appropriate asymptotic behavior of $h_{l, \sigma}^{G}(r)$, we take its asymptotic limit

$$
h_{l, \sigma}^{G}(r) \longrightarrow A_{l}^{R e g} \sin \left(\Phi_{C}(l)+\sigma_{C}(l)\right)+a_{l, \sigma} \mathscr{N}_{\text {source }} \cos \left(\Phi_{C}(l+\sigma+2)+\delta(l, \sigma, \lambda)\right) .
$$

By separating the incoming and outgoing waves, the previous equation can be re-written as follows

$$
\begin{aligned}
h_{l, \sigma}^{G}(r) \longrightarrow \frac{1}{2}( & \left.a_{l, \sigma} \mathscr{N}_{\text {source }} e^{i \delta_{l, \sigma, \lambda}}-i A_{l}^{R e g} e^{i \sigma_{C}(l)}\right) e^{i \Phi_{C}(l)} \\
& +\frac{1}{2}\left(a_{l, \sigma} \mathscr{N}_{\text {source }} e^{-i \delta_{l, \sigma, \lambda}}+i A_{l}^{R e g} e^{-i \sigma_{C}(l)}\right) e^{-i \Phi_{C}(l)}
\end{aligned}
$$

where

$$
\delta_{l, \sigma, \lambda}=\delta(l, \sigma, \lambda)-\frac{\pi}{2}(\sigma+2) .
$$

Hence, to provide the general solution

$$
h_{l, \sigma}^{G}(r)=A_{l}^{\operatorname{Reg}} v_{l}^{\operatorname{Reg}}(r)+h_{l, \sigma}^{P}(r),
$$


with a pure incoming $(+)$ or outgoing $(-)$ behavior $\left(e^{ \pm i \Phi_{C}(l)}\right)$, the constants $A_{l}^{\text {Reg }}$ and $a_{l, \sigma}$ of the source must be chosen as

$$
A_{l}^{\text {Reg } \pm}= \pm i \frac{e^{\mp i \delta_{l, \sigma, \lambda}}}{\cos \left(\delta_{l, \sigma, \lambda}-\sigma_{C}(l)\right)}, \quad a_{l, \sigma}^{ \pm}=\frac{1}{\mathscr{N}_{\text {source }}} \frac{e^{\mp i \sigma_{C}(l)}}{\cos \left(\delta_{l, \sigma, \lambda}-\sigma_{C}(l)\right)},
$$

while, for standing wave cosine-like behavior $\cos \left(\Phi_{C}(l)+\sigma_{C}(l)\right)$,

$$
A_{l}^{\text {Reg }}=\tan \left(\delta_{l, \sigma, \lambda}-\sigma_{C}(l)\right), \quad a_{l, \sigma}=\frac{1}{\mathscr{N}_{\text {source }} \cos \left(\delta_{l, \sigma, \lambda}-\sigma_{C}(l)\right)} .
$$

In Fig. 1, we plot as a function of $r$ the solution $h_{l, \sigma}^{G}(r)$ (the $\varphi_{c}(r)$ of Ref. [8]) constructed to have standing wave cosine-like behavior, for $l=0$ and $l=1$. For the illustration, we elected the following parameters: $\mu=1, z_{1} z_{2}=-1, k=1, \lambda=0.5$ and $\sigma=0$. This is the case considered by Yamani and Reinhardt [8] and besides is the one used in J-matrix calculations nowadays (see, e.g., [20]). We plot also the non-homogeneity $e^{-\lambda r}$, and the sought after cosine-like behavior $\cos \left(\Phi_{C}(l)+\sigma_{C}(l)\right)$. It is clear that this asymptotic behavior is reached by the function $h_{l, \sigma}^{G}(r)$ only in a region where the exponential vanishes. This, however, depends on the parameters $\lambda, l$ and $\sigma$; for example, the cosine-like behavior is reached further away for $l=1$ than for $l=0$.

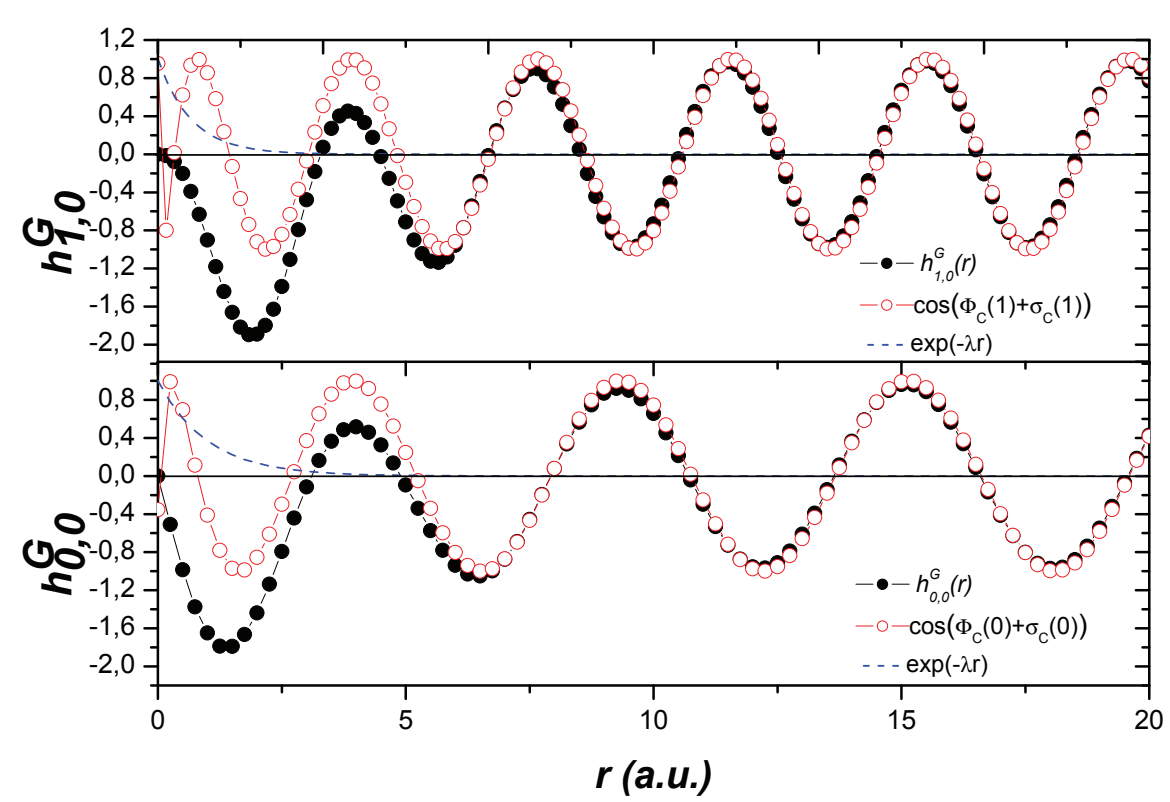

Figure 1: The function $h_{l, \sigma}^{G}(r)$ constructed to have standing wave cosine-like behavior is plotted (solid circles) as a function of $r$ for $l=0,1$, and compared to the cosine-like behavior $\cos \left(\Phi_{C}(l)+\sigma_{C}(l)\right)$ (open circles). The parameters used are: $\mu=1, z_{1} z_{2}=-1, k=1, \lambda=0.5$ and $\sigma=0$. The exponential $e^{-\lambda r}$ (dashed line) is also included for comparison. 


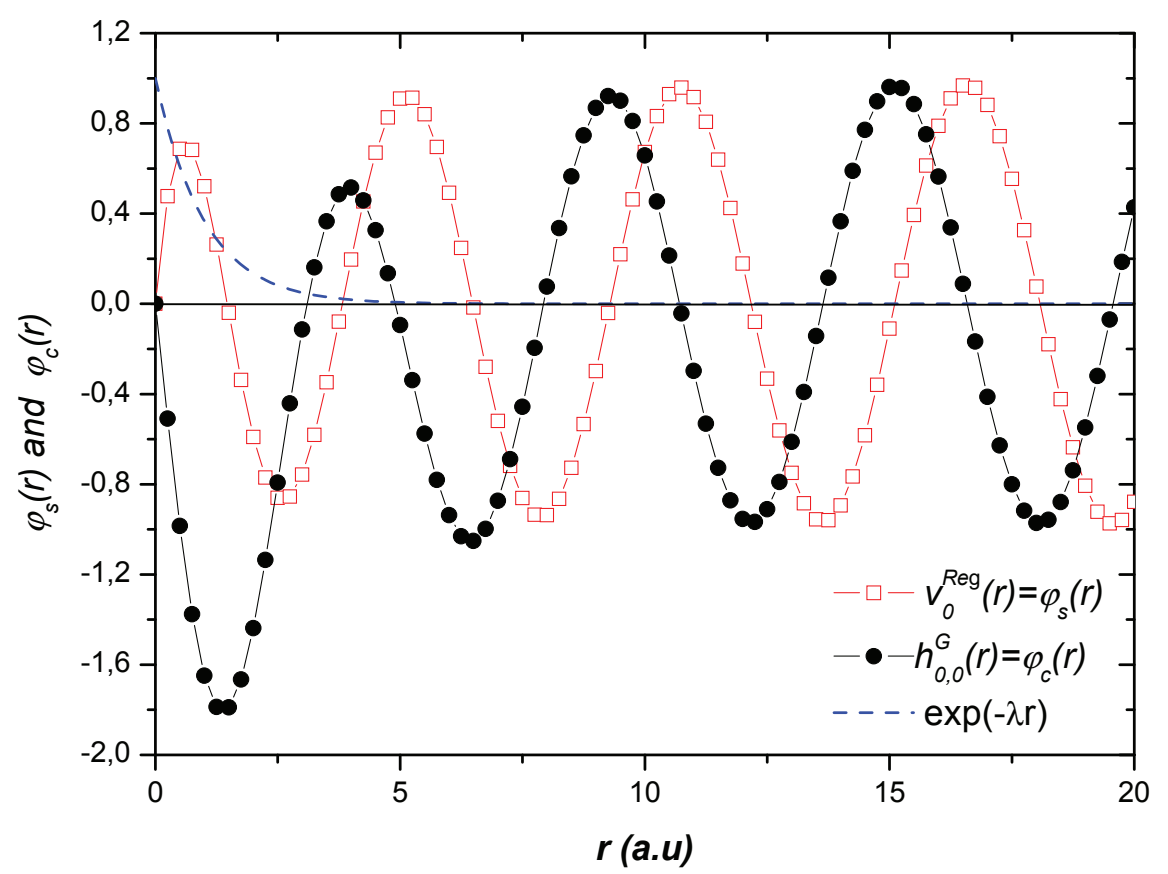

Figure 2: The function $h_{l, \sigma}^{G}(r)$ constructed to have standing wave cosine-like behavior is plotted (solid circles) as a function of $r$ for $l=0$, and compared to $v_{l}^{R e g}(r)$ (open squares) possessing the sine-like behavior $\sin \left(\Phi_{C}(l)+\sigma_{C}(l)\right)$ associated to the homogeneous solution. The parameters used are: $\mu=1, z_{1} z_{2}=-1$, $k=1, \lambda=0.5$ and $\sigma=0$. The exponential $e^{-\lambda r}$ (dashed line) is also included for comparison.

In Fig. 2 we compare the functions $h_{l, \sigma}^{G}(r)$ (the $\varphi_{c}(r)$ of Ref. [8]) and $v_{l}^{\text {Reg }}(r)$ (the $\varphi_{s}(r)$ of Ref. [8]). The two solutions become linearly independent, only asymptotically, i.e., in the region where $e^{-\lambda r}$ becomes negligible. This linear independency, however, depends upon the parameters $\lambda, l$ and $\sigma$. Other values of $\lambda$ and $\sigma$ have been considered and lead to similar observations; for increasing values of $\lambda$, i.e., a source of shorter range, the convergence to a cosine-like behavior is faster as one would expect.

Finally, it is interesting to consider the situation where the Coulomb potential is absent, $z_{1} z_{2}=0$. For the same non-homogeneity, the particular solution of the non-homogeneous Schrödinger equation (1) is given by Eq. (3) with $\alpha=0$. This case has been considered in, e.g., Refs. $[7,8,11]$, within the context of a $L^{2}$ representation of scattering functions and J-matrix approaches for short-range potentials. The regular and irregular homogeneous solutions reduce to spherical Bessel functions, and behave at large distances as [18]

$$
\begin{aligned}
& v_{l}^{\text {Reg }}(r) \longrightarrow \sin \left(k r-\frac{\pi}{2} l\right), \\
& v_{l}^{\text {Irreg }}(r) \longrightarrow \cos \left(k r-\frac{\pi}{2} l\right) .
\end{aligned}
$$


In this case, the conditions to obtain a pure incoming $(+)$ or outgoing $(-)$ behavior are

$$
A_{l}^{R e g \pm}= \pm i\left[1 \mp \tan \left(\delta_{l, \sigma, \lambda}\right)\right], \quad a_{l, \sigma}^{ \pm}=\frac{1}{\mathscr{N}_{\text {source }}} \frac{1}{\cos \left(\delta_{l, \sigma, \lambda}\right)},
$$

while, for standing wave cosine-like behavior,

$$
A_{l}^{\text {Reg }}=\tan \left(\delta_{l, \sigma, \lambda}\right), \quad a_{l, \sigma}=\frac{1}{\mathscr{N}_{\text {source }} \cos \left(\delta_{l, \sigma, \lambda}\right)},
$$

where

$$
\delta_{l, \sigma, \lambda}=-\arg \left[{ }_{2} F_{1}\left(\begin{array}{c|c}
\sigma+1,2 l+2+\sigma \\
l+2+\sigma
\end{array} \mid ; \frac{1}{2}\left(1+\frac{\lambda}{i k}\right)\right)\right]-\frac{\pi}{2}(\sigma+2) .
$$

\section{Summary}

In the present contribution we have provided a closed form solution for a radial Coulomb nonhomogeneous Schrödinger equation used within the J-matrix approach (the free-particle, corresponding to $z_{1} z_{2}=0$, was also considered as a subcase). The particular solution is expressed in terms of a two-variable hypergeometric function, and presents a cosine-like asymptotic behavior at large distances. When compared with the cosine-like behavior associated to the homogeneous solution, a phase-shift depending on the source parameters appears. An appropriate linear combination of the homogeneous and the particular solution was found in such a way to obtain functions having pure incoming, outgoing and standing-wave cosine-like behavior with Coulombic phase. All this functions are real and regular at the origin.

The results presented in this article can be used for solving scattering problems within the J-matrix or alternative variational methods [13]. Besides, the present results and those of Ref. [6] are important for studying the details of a formulation such as the exterior complex scaling $[3,5]$. This method is based on the solution of a non-homogeneous equation as the one considered here where an artificial cut-off is introduced on the source term. How this cut-off affects the solution of the scattering problem is the object of our current investigations.

Acknowledgments. One of the authors (G. Gasaneo) thanks the support by PGI (24/F049) of the Universidad Nacional del Sur, of ANPCyT (PICT08/0934) (Argentina) and PIP 200901/552 of CONICET (Argentina). The support provided by the Lorraine region for the visit of the Dr. Gasaneo to the Laboratoire de Physique Moléculaire et des Collisions, Université Paul VerlaineMetz is also gratefully acknowledged.

\section{References}

[1] R. G. Newton, Scattering Theory of Waves and Particles (Dover Publications INC, New York, 2002).

[2] C. J. Joachain, Quantum Collision Theory (North-Holland Publishing Company, 1983). 
[3] T. N. Rescigno and C. W. McCurdy, Phys. Rev. A 55 (1997) 4253.

[4] T. N. Rescigno, M. Baertschy, W. A. Isaacs, and C. W. McCurdy, Science 286 (1999) 2474.

[5] C. W. McCurdy, M. Baertschy, and T. N. Rescigno, J. Phys. B: At. Mol. Opt. Phys. 37 (2004) R137.

[6] G. Gasaneo and L. U. Ancarani, Phys. Rev. A 82 (2010) 042706

[7] A. D. Alhaidari, E. J. Heller, H. A. Yamani, and M. S. Abdelmonem, The J- Matrix Method, Development and Applications (Springer, Berlin, 2008).

[8] H. A. Yamani and W. P. Reinhardt, Phys. Rev. A 11 (1975) 1144.

[9] J. T. Broad, Phys. Rev. A 31 (1985) 1494.

[10] E. J. Heller and H. A. Yamani, Phys. Rev. A 9 (1974) 1201.

[11] H. A. Yamani and L. Fishman, J. Math. Phys. 16 (1975) 410.

[12] J. T. Broad and W. P. Reinhardt, J. Phys. B: At. Mol. Opt. Phys. 9 (1976) 1491.

[13] S. K. Adhikari, Variational Principles and the Numerical Solution of Scattering Problems (JohnWiley and Sons, New York, 1998).

[14] L. D. Landau and E. M. Lifshitz, Quantum Mechanics: Non-Relativistic Theory (Pergamon, Oxford, 1965).

[15] L. U. Ancarani and G. Gasaneo, J. Math. Phys. 49 (2008) 063508.

[16] A. Maquet, V. Veniard, and T. A. Marian, J. Phys. B: At. Mol. Opt. Phys. 31 (1998) 3743.

[17] G. Gasaneo and L. U. Ancarani, Phys. Rev. A 80 (2009) 062717.

[18] M. Abramowitz and I. A. Stegun, Handbook of Mathematical Functions (Dover, New York, 1972).

[19] L. U. Ancarani and G. Gasaneo, J. Phys. A: Math. Theor. 43 (2010) 085210.

[20] E. Foumouo, G. L. Kamta, G. Edah, and B. Piraux, Phys. Rev. A 74 (2006) 063409. 